\title{
Plasma deposition of thin layers containing titanium and barium with the use of DBD*
}

\author{
Małgorzata Majdaka , Teresa Opalińska, Bartłomiej Wnęk, and Piotr Konarski \\ Tele \& Radio Research Institute, 00-241 Warsaw, 44/50 Długa str., Poland
}

Received: 30 September 2012 / Received in final form: 6 December 2012 / Accepted: 19 December 2012 Published online: 15 February 2013 - (c) The Author(s) 2013

\begin{abstract}
Two different methods of deposition of thin layers containing titanium and barium with the use of dielectric barrier discharge were investigated. The first was carried out in two stages. The first stage consisted in transferring compounds containing titanium and barium from the vapor of the organic precursor to the copper substrate, while the second stage involved the etching of the organic layer in oxygen. The second method consisted in transferring titanium and barium to the copper substrate from the ceramic plate made of barium titanate, which was one of the electrodes during the plasma process. The results of analysis obtained by SIMS showed directly that both methods gave a positive result in the deposition of the thin layers containing a certain amount of titanium and barium on the surface of the copper plate. However, more effective method was that involving the properties of reaction with the metallorganic precursor. FTIR study of the layers prepared from the organic precursor indirectly showed that layers deposited on the copper plate contained compounds of barium and titanium. FTIR study also revealed that during the process of etching in oxygen, the organic layer was decreased, but it was not completely removed.
\end{abstract}

\section{Introduction}

A large number of works are dedicated to studies of the most widely applied ferroelectric material which nowadays is barium titanate.

Materials such as $\mathrm{BaTiO}_{3}$, due to their high dielectric constant [1-8], are commonly used in microelectronic devices such as multilayer ceramic capacitors [1,4-12], random-access memories (RAM) $[2,4,5,7,11,12]$, optoelectronic devices $[1,5,7,10]$ or sensors $[6,13,14]$.

The miniaturization of those microelectronic devices requires reducing the size of their building materials $[3,9]$, which should be characterized by high degree of crystallization and high smoothness [3]. For this reason, nowadays the interest in receiving the high quality of various nanolayers has been growing. Furthermore, thin layers have additional advantages in comparison with thick films - it is a higher repeatability in manufacturing process and a better control of fabrication parameters [14].

So far, applied methods of preparing $\mathrm{BaTiO}_{3}$ thin layers such as sol-gel $[1,7,15,16]$, sputtering [7], conventional furnace sintering [6] or assisted chemical vapor deposi-

\footnotetext{
* Contribution to the Topical Issue "13th International Symposium on High Pressure Low Temperature Plasma Chemistry (Hakone XIII)", Edited by Nicolas Gherardi, Henryca Danuta Stryczewska and Yvan Ségui.

a e-mail: malgorzata.majdak@itr.org.pl
}

tion (for example, RF-CVD) [7,9,11] required using high temperatures, usually above $500{ }^{\circ} \mathrm{C}[5,7,11]$. However, the thermal stress often results in cracking or peeling of the deposited thin films [7,11]. Some of previously applied methods require the use of substrate, that contains one of the compounds of $\mathrm{BaTiO}_{3}$. For example: in the hydrothermal-electrochemical method in which $\mathrm{BaTiO}_{3}$ layer was deposited on the substrate made of titanium sheets [7] or in the electrochemical deposition used by Zeng et al. who used the TiN-coated Si substrate [11].

In this paper, two alternative methods of preparing thin layer containing barium and titanium are studied. The new methods, based on the low-temperature plasma processes using dielectric barrier discharge, do not require the use of high temperature or the substrate that would contain one of the compounds of $\mathrm{BaTiO}_{3}$ thin layer.

The first of the presented methods (method I) was based on transferring the titanium and barium ions from tetrahydrofurfuryl barium and titanium (IV) oxide $\left(\mathrm{C}_{30} \mathrm{H}_{54} \mathrm{BaO}_{12} \mathrm{Ti}\right)$ - the organic precursor to the copper substrate. So far, the most commonly used organic precursors of those elements were $\mathrm{Ba}\left(\mathrm{CH}_{3} \mathrm{COO}\right)_{2}$ (barium acetate) $[1,11,17]$ and $\mathrm{Ti}(\mathrm{OiPr})_{4}$ (titanium tetra-isopropoxide) $[1,4,18]$. In this paper we use only one metallorganic precursor, that significantly simplifies the experimental setup. 


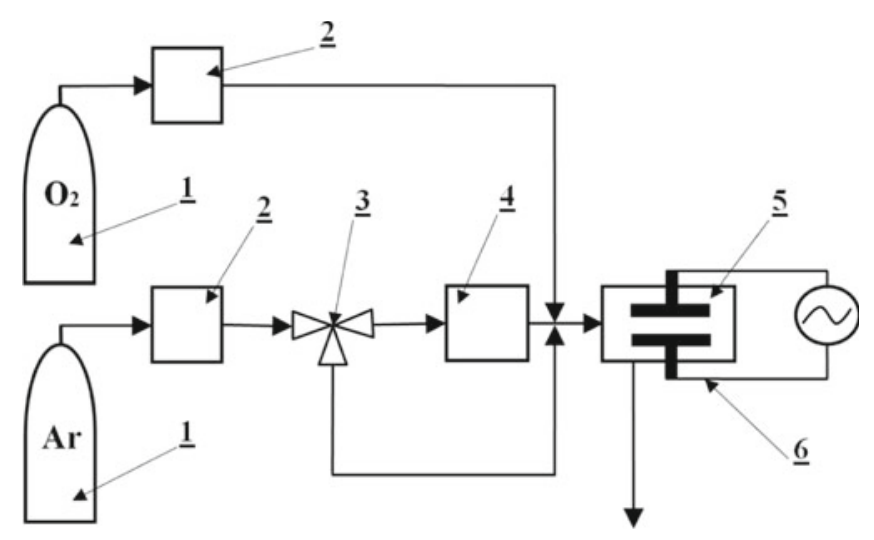

Fig. 1. Scheme of the experimental setup; (1) a working gas vessel, (2) mass flow controllers, (3) a shuttle valve, (4) a bubbling washer, (5) a plasma reactor, (6) a power supply.

The second presented method (method II) consisted in transferring the compounds of titanium and barium from a ceramic plate made of barium titanate to the copper substrate.

In this paper we present the study of preparing thin layers containing barium and titanium in the most simplified experimental setup. This simplicity was obtained by using the dielectric barrier discharge generated under the atmospheric pressure at the ambient temperature, a multicomponent organic oxide - only one precursor of all compounds of the desired thin layer and ferroelectric ceramics as the other precursor.

\section{Experimental section}

A scheme of the experimental setup is shown in Figure 1.

The experimental setup consisted of: a working gas vessel (argon or oxygen), a mass flow controllers, a bubbling washer with the organic precursor, a plasma reactor and the power supply. Argon (99.999\%) flow rate amounted to $0.06-1.10 \mathrm{NL} / \mathrm{min}$ and that of oxygen (99.999\%) 1.67-2.0 NL/min. Experiments were carried out in 20-60 min. The discharge was generated with frequency of $50 \mathrm{kHz}$ and the voltage amounted to $0.57-1.75 \mathrm{kV}$.

Thin films were prepared by means of two methods method I and method II. The first one (method I) consisted in preparing a thin layer containing titanium and barium with the use of precursor of $\mathrm{Ti}^{4+}$ and $\mathrm{Ba}^{2+}$ ions. The precursor was the organometallic compound - tetrahydrofurfuryl barium and titanium (IV) oxide $\left(\mathrm{C}_{30} \mathrm{H}_{54} \mathrm{BaO}_{12} \mathrm{Ti}\right.$ solution with tetrahydrofururyl alcohol and ethanol - Sigma-Aldrich).

The method I was performed in two ways. The first way consisted of two stages. In the first stage, the working gas (argon) flowing through the bubbling washer with the precursor washed away the organometallic compound and delivered it to the discharge zone. There was a discharge generated between the metal (copper) surface of the high-voltage electrode and the surface of the dielectrics mounted to the grounded electrode. In the second stage,
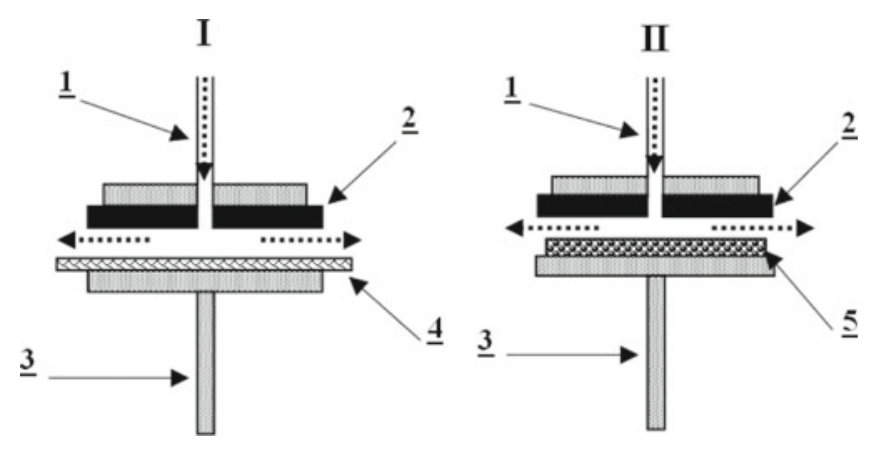

Fig. 2. Scheme of electrodes construction in reaction with the precursor (method I) and in reaction with the ferroelectric ceramics (method II); (1) high-voltage electrode/a working gas inlet; (2) copper plate (substrate); (3) grounded electrode; (4) dielectric barrier; (5) ferroelectric ceramics.

the obtained layer was etched in the discharge generated in the atmosphere of a mixture of argon and oxygen. The second way consisted of only one stage (performed for Cu6 sample). The working gas (argon) flowing through the bubbling washer washed away the molecules of the precursor. The stream of argon with the vapor of the precursor was mixed with oxygen after the bubbling washer, but before the plasma reactor. Mixed components of the reaction were delivered to the discharge zone, which was the same as in the previously mentioned process. A few additional materials were used as the dielectric barriers. They were: polyimide (PI), polycarbonate (PC) and poly(methyl methacrylate) (PMMA).

The second method (method II) consisted in generating the discharge in argon between the high-voltage copper electrode and the grounded electrode made of $\mathrm{BaTiO}_{3}$. Figure 2 presents schemes of the electrodes constructions used in each of the described methods.

Two different methods were used to analyze the obtained layers: the infrared spectroscopy with Fourier transformation (FTIR) and the secondary ion mass spectrometry (SIMS). The research carried out by FTIR was performed by means of Nicolet FTIR spectrometer (ThermoScientific IS10 company), equipped with the ATR (attenuated total reflectance - the method of total internal reflection) with a ZnSe crystal. Number of scans was 64 , the spectra were measured with resolution of $4 \mathrm{~cm}^{-1}$. The research carried out by SIMS was performed using secondary ion mass spectrometer - SAJW-05 [19]. The etching with $\mathrm{Ar}^{+}$ions was performed with the energy of $3 \mathrm{keV}$. The depth profile analyses were performed at 0-3 $\mu$ depth range and additionally at $0-100 \mathrm{~nm}$ depth range. The depth of the resulting craters was measured with the stylus profilometer Alpha-step 100 Tencor.

\section{Results and discussion}

\subsection{Theoretical assumptions for the methods}

For the method I, it was assumed that the working gas (argon) flowing through the bubbling washer with 
the precursor would wash away the vapor of the organometallic compound and would introduce it to the discharge zone. The large organic molecule would be decomposed in the argon plasma. Barium and titanium in the form of atoms, oxides or partially chemically bounded in the organic phase would be deposited on the surfaces of both electrodes. Whereas the part of the precursor that contains carbon, oxygen and hydrogen would be decomposed in the argon plasma to light organic compounds and would be removed with the working gas out of the discharge zone.

The preliminary FTIR analysis showed that during the plasma reaction, mainly the organic layer is deposited on the copper substrate. Therefore, the method I was supplemented with the additional stage - etching the obtained organic layer in the oxygen plasma. Ozone, oxygen radicals and excited molecules of oxygen formed in the discharge would remove or decrease the organic layer. Furthermore, during the reaction, oxygen molecules would form the chemical structure of barium titanite or titanium oxides on the surface of substrate. The mixture of argon and oxygen was applied to excite plasma easier.

For the method II, it was assumed that plasma affecting the surface of the ferroelectric plate would transfer the compounds containing barium and titanium from the ceramics to the surface of the copper substrate.

\subsection{SIMS analysis}

Figure 3a presents the results of SIMS depth profile analysis of the samples $\mathrm{Cu} 1, \mathrm{Cu} 2, \mathrm{Cu} 3, \mathrm{Cu} 4$ and $\mathrm{Cu} 6$. The ${ }^{12} \mathrm{C}^{+}$, ${ }^{48} \mathrm{Ti}^{+}$and ${ }^{138} \mathrm{Ba}^{+}$secondary ion currents are normalized versus ${ }^{63} \mathrm{Cu}^{+}$secondary ion current. Plots of $\mathrm{Cu} 1$ and $\mathrm{Cu} 2$ samples show significant contribution of carbon presence in the sputtering range corresponding to $600 \mathrm{~nm}$ and $3 \mu \mathrm{m}$ depth range respectively. Plots of $\mathrm{Cu} 3$ and $\mathrm{Cu} 4$ samples show higher contribution of titanium with respect to $\mathrm{Cu} 1$ and $\mathrm{Cu} 2$ samples.

Both $\mathrm{Cu} 1$ and $\mathrm{Cu} 2$ samples contain a layer of carbon. The carbon deposited on the surface of the copper substrate comes from the organic layer, which was formed during the plasma process carried out by means of the method I. For these two samples, only the first stage of the method I was performed. It seems that the organic layer on the surface of the Cu1 sample fully covers the copper substrate and is dense in comparison with that of the $\mathrm{Cu} 2$ sample, on which the organic layer is thicker, but also more porous, so the other analyzed elements may be seen through it. The Cu3 sample was also treated with the method I, but in this case, both stages of the process were completed - the reaction with the precursor and plasma etching in oxygen was performed.

The organic coating of the Cu3 sample is much thinner in comparison with those of the $\mathrm{Cu} 1$ and $\mathrm{Cu} 2$ samples, which indicates that the plasma reaction in oxygen resulted in partial etching of this layer. The $\mathrm{Cu} 4$ sample was treated in argon with the use of the method II, in the discharge with the ceramic barrier. The layers on the $\mathrm{Cu} 3$ and $\mathrm{Cu} 4$ samples contain a lot more of titanium in com-

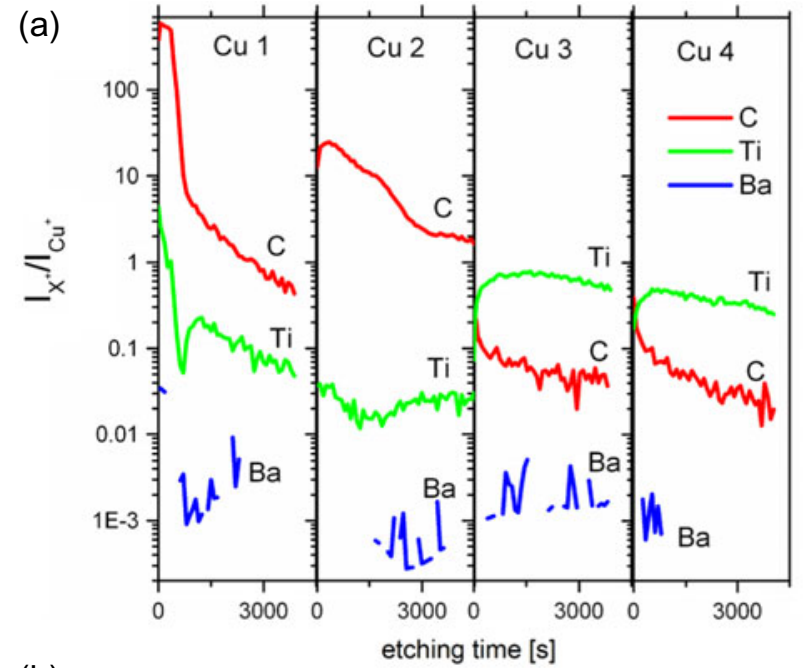

(b)
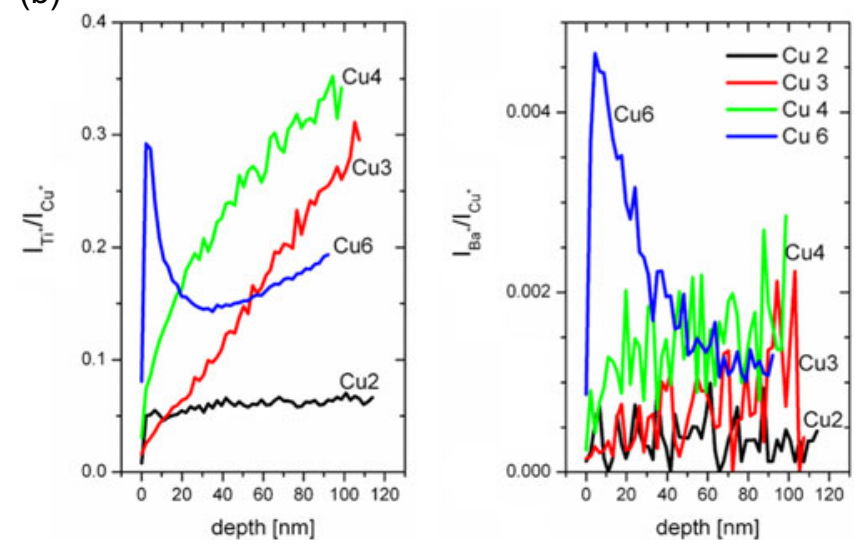

Fig. 3. The results of SIMS depth profile analysis presented as ion currents of ${ }^{12} \mathrm{C}^{+},{ }^{48} \mathrm{Ti}^{+}$and ${ }^{138} \mathrm{Ba}^{+}$divided by ${ }^{63} \mathrm{Cu}^{+}$ secondary ion current: (a) values are plotted versus the etching time (sputtering rate $1.5 \mathrm{~nm} / \mathrm{s}$ ) and (b) versus the depth of etching (sputtering rate $0.05 \mathrm{~nm} / \mathrm{s}$ ).

parison with the layer on the $\mathrm{Cu} 2$ sample. The samples $\mathrm{Cu} 1-\mathrm{Cu} 4$ contain a small amount of barium.

The additional SIMS study was performed to analyze the profile in the range of $0-100 \mathrm{~nm}$ (Fig. 3b). In this analysis three secondary ion currents of ${ }^{63} \mathrm{Cu}^{+},{ }^{48} \mathrm{Ti}^{+}$and ${ }^{138} \mathrm{Ba}^{+}$were examined. Four samples were analyzed: $\mathrm{Cu} 2$, $\mathrm{Cu} 3, \mathrm{Cu} 4$ and $\mathrm{Cu} 6$.

The Cu6 sample shows the presence of a thin layer on the copper substrate. This layer is about $20 \mathrm{~nm}$ thick and is enriched in titanium and barium. The distribution of these two elements is different than in the samples $\mathrm{Cu} 2$, $\mathrm{Cu} 3$ and $\mathrm{Cu} 4$. The $\mathrm{Cu} 3$ and $\mathrm{Cu} 4$ samples show wide distribution of titanium and barium in the range to $120 \mathrm{~nm}$, without enrichment in the range of $20 \mathrm{~nm}$ depth.

The results of SIMS show that the most promising process was this performed for the sample Cu6. The layer for this sample was obtained by the method I (Tab. 1). The reaction conditions for this sample were different than those for the other samples. A higher argon flow rate was applied, therefore larger amount of the precursor was transferred to the discharge zone. In addition, oxygen was 
Table 1. The experimental parameters.

\begin{tabular}{lcccc}
\hline Sample & Method/stage & $\begin{array}{c}\mathrm{VAr} \\
{[\mathrm{NL} / \mathrm{min}]}\end{array}$ & $\begin{array}{c}\mathrm{VO}_{2} \\
{[\mathrm{NL} / \mathrm{min}]}\end{array}$ & Dielectric \\
\hline $\mathrm{Cu} 1$ & $\mathrm{I} / 1$ & 0.06 & - & $\mathrm{PC}$ \\
$\mathrm{Cu} 2$ & $\mathrm{I} / 1$ & 0.28 & - & $\mathrm{PMMA}$ \\
$\mathrm{Cu} 3$ & $\mathrm{I} / 1+2$ & 0.14 & 1.67 & $\mathrm{PI}$ \\
$\mathrm{Cu} 4$ & $\mathrm{II}$ & 0.20 & - & $\mathrm{BaTiO}_{3}$ \\
$\mathrm{Cu} 5$ & $\mathrm{I} / 1$ & 0.12 & - & $\mathrm{PI}$ \\
$\mathrm{Cu} 6$ & $\mathrm{I}^{*}$ & 1.10 & 2.00 & $\mathrm{PMMA}^{*}$ \\
\hline only one stage - reaction in flow of mixed $\mathrm{Ar}$ and $\mathrm{O}_{2}$.
\end{tabular}

added to the reaction mixture in one stage with argon. This enabled the reaction between oxygen and the precursor in the gaseous phase, before depositing the organometallic molecules on the copper surface. However, in the reaction with two stages, as was in the case of the preparation of layer on the $\mathrm{Cu} 3$ sample, oxygen in the second stage reacted with the precursor already deposited on the surface of the substrate. The reaction runs easier in the gaseous phase, and additionally the total gas flow was significantly higher for the $\mathrm{Cu} 6$ than in the case of the preparation of the layers on the other samples.

Therefore in the reaction of the $\mathrm{Cu} 6$ sample it was more likely to: (1) reduce the amount of the organic layer deposited on the substrate and (2) increase the quantity of barium and titanium deposited on the surface.

The presented results improve the positive effect of the surface modification in the plasma and confirm the effectiveness of the method in the process of depositing a thin layer containing titanium and barium on the chosen substrate. Basing on these results, it can be concluded, that both, method I and method II, gave a positive result in the deposition of the thin layer containing certain amounts of $\mathrm{Ti}$ and $\mathrm{Ba}$ on the copper substrate. However, the method I, which was performed in only one stage (mixed stream of oxygen and argon), was more effective.

\subsection{FTIR analysis}

FTIR was used to determine the composition of the organic layer obtained on the copper substrate during the experiment carried out with the use of method I. The Cu5 sample - the sample after processing with the use of the first stage of the method I and the Cu3 sample the samples after processing with the use of both stages of method I and the Cu6 sample - the sample after processing in mixed stream of argon and oxygen in the method I were examined. The organic layer formed on the copper substrate should contain functional groups that are present in the precursor solution and possibly groups of derivatives, which could be formed from the decomposition of the specific compounds. Figures 4 and 5 present the IR spectra of ethyl alcohol and tetrahydrofurfuryl alcohol - the solutions in the precursors mixture, whose IR spectra are shown in Figure 6. For a comparison, Figure 7 presents the spectra of the organic layers that were ob-

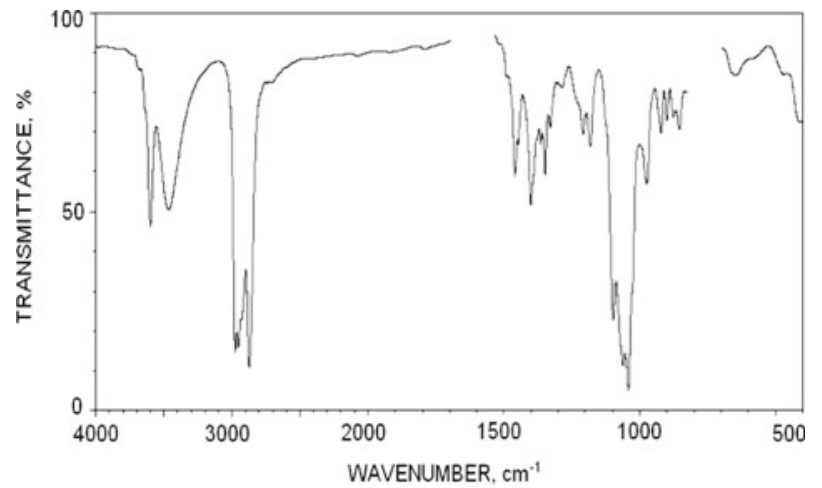

Fig. 4. The IR spectrum of tetrahydrofurfuryl alcohol [20].

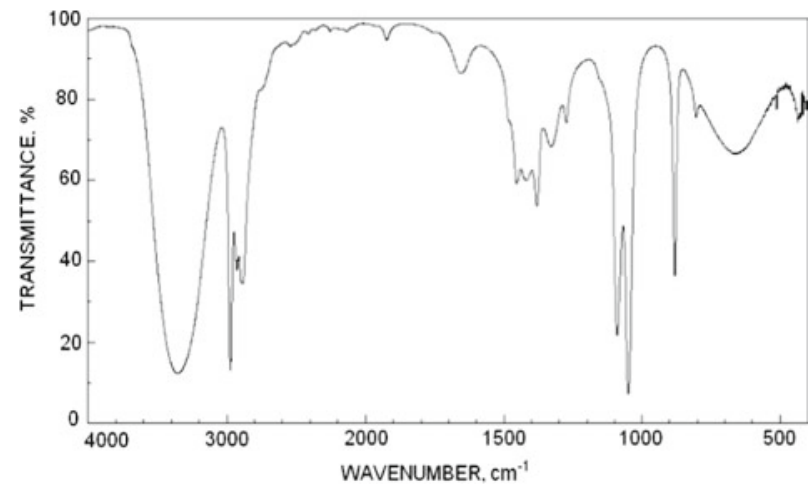

Fig. 5. The IR spectrum of ethyl alcohol [20].

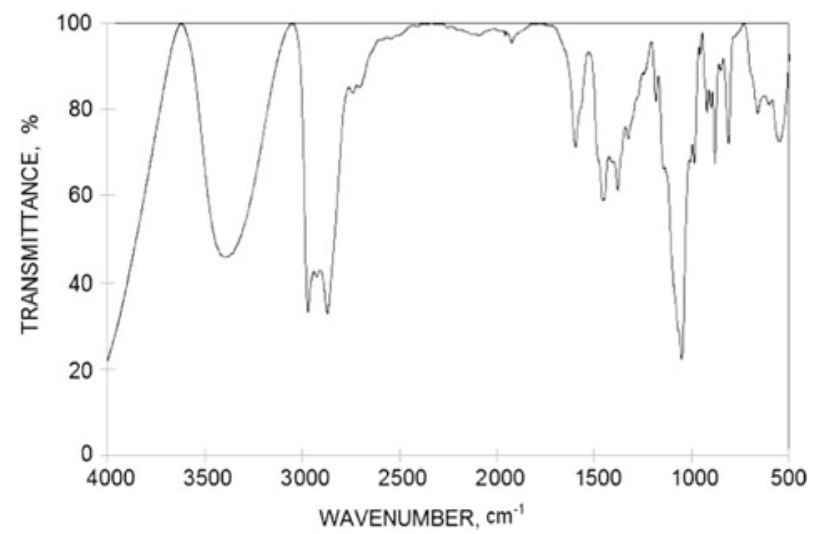

Fig. 6. The IR spectrum of the precursors $\left(\mathrm{C}_{30} \mathrm{H}_{54} \mathrm{BaO}_{12} \mathrm{Ti}\right)$ solution in tetrahydrofurfuryl alcohol and ethanol.

tained on a copper substrate by means of the method I (Tab. 1).

The spectrum of tetrahydrofurfuryl barium and titanium oxide (precursor) looks similarly to the spectrum of tetrahydrofurfuryl alcohol - solvent, since both compounds are structurally similar and have the same functional groups, excluding the $\mathrm{OH}$ group, which is not present in the precursor molecule. Comparing all the presented spectra, it is easy to see that they have some common features - the main group at the same wavenumbers. Each of these spectra contains a group of $\sim 3500 \mathrm{~cm}^{-1}$, which corresponds to the $\mathrm{OH}$ group present in alcohol. The other similar features are: $2800-2900 \mathrm{~cm}^{-1}$ signals, 
M. Majdak et al.: Plasma deposition of thin layers containing titanium and barium with the use of DBD
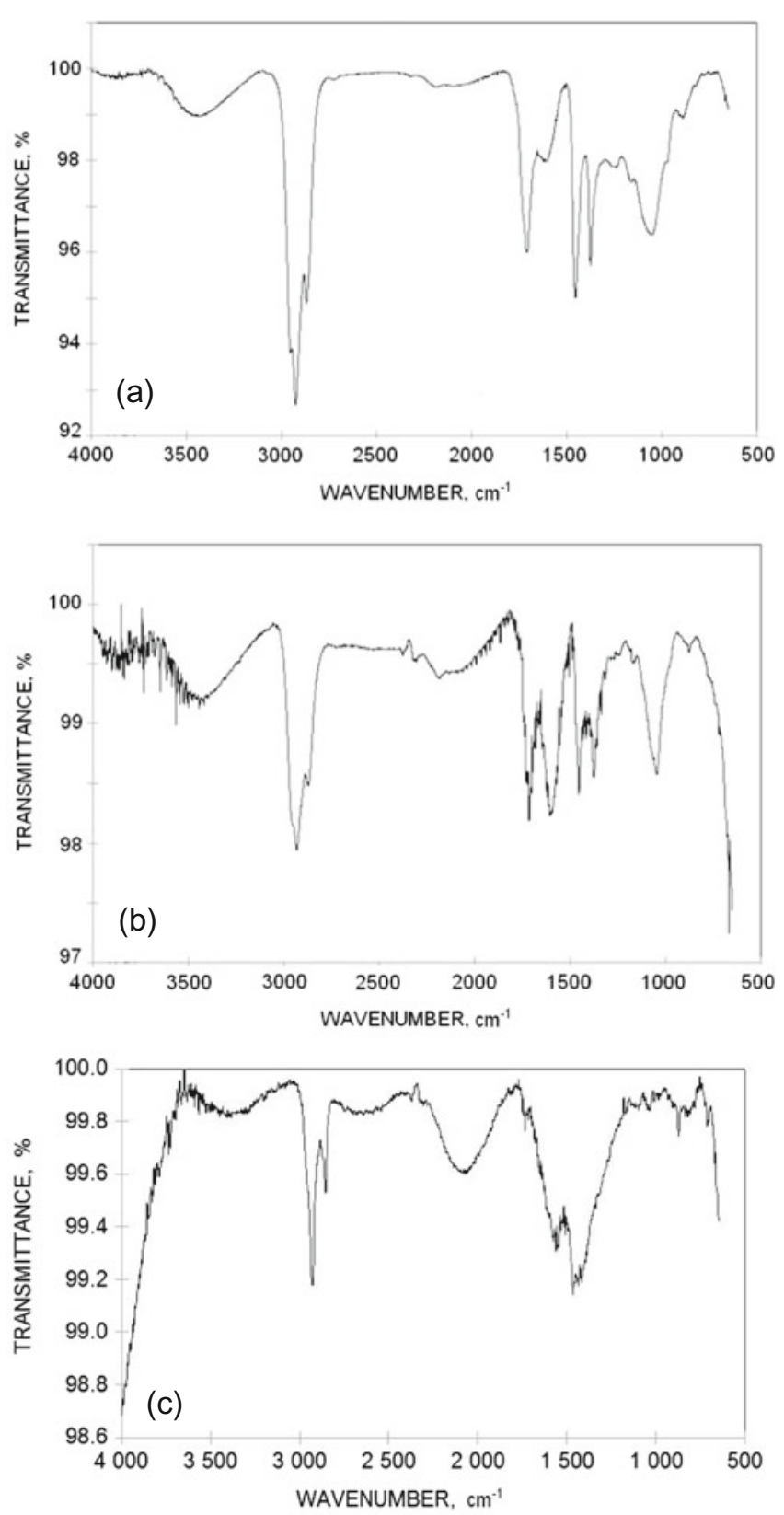

Fig. 7. The IR spectra of the layers obtained on the copper substrate: (a) the sample after processing with the use of the first stage of the method I - Cu5, (b) the sample after processing with the use of both stages of method I- Cu3 and (c) the samples after processing in mixed stream of argon and oxygen in the method I - Cu6.

that correspond to the aliphatic $\mathrm{CH}$ carbon atoms that are not bound with unsaturated bonds. Groups occurring at $1400-1500 \mathrm{~cm}^{-1}$ could not be identified on the basis of tables of specific bonds. However, these are probably the groups corresponding with the unsaturated C-C bonds. The other similar features are $1000-1100 \mathrm{~cm}^{-1}$ signals, that correspond to $\mathrm{CO}$ groups in primary alcohols (and ethers). It is supposed that this group is higher for tetrahydrofurfuryl alcohol (Fig. 4), because it contains the oxygen bound in the ether group COC in its molecule. Similar in shape is the spectrum of the Cu5 sample (Fig. 7a), that may indicate the presence of ether groups in the surface layer of the sample.

The new groups for wavenumbers $\sim 1600-1700 \mathrm{~cm}^{-1}$, which are not present in the spectrum of any of the solvents, were in the spectrum of the layers obtained in plasma (Fig. 7) and in the spectrum of the precursors solution (Fig. 6).

To identify these groups we had to refer to the example of two compounds similar in structure: ethyl alcohol $\left(\mathrm{C}_{2} \mathrm{H}_{5} \mathrm{OH}\right)$ and the other in which hydrogen atom is replaced with sodium $\left(\mathrm{C}_{2} \mathrm{H}_{5} \mathrm{ONa}\right)$ [20]. It was noticed that the $\mathrm{C}_{2} \mathrm{H}_{5} \mathrm{ONa}$ spectrum included additional groups for wavenumbers $\sim 1600-1700 \mathrm{~cm}^{-1}$. The signal at this point probably corresponds with $\mathrm{CO}$ combined with oxygen, which is bonded ionically with sodium cation. That caused a shift of this signal toward the higher wavenumbers. A similar situation occurs when we consider the structure of the precursor and tetrahydrufurfuryl alcohol. The precursor cations $\mathrm{Ba}^{2+}$ and $\mathrm{Ti}^{4+}$ are bonded to the oxygen atom. A similar oxygen atom in the molecule is related to the hydrogen atom in the solvent molecule. Thus, the spectrum of the precursor contains the additional spectrum with the wavenumber $\sim 1600-1700 \mathrm{~cm}^{-1}$. This group is also observed in the spectrum of the layers obtained on the copper substrates (Fig. 7), indicating that the precursor had evaporated from the bubbling washer during the process and was deposited on the copper substrate. The presence of oxidized groups corresponding with the cations $\mathrm{Ba}^{2+}$ and $\mathrm{Ti}^{4+}$ indicates the presence of these cations in the examined layer.

Comparing the spectrum of the $\mathrm{Cu} 5$ (Fig. 7a), Cu3 (Fig. 7b) and Cu6 (Fig. 7c) samples, we can see that they are similar. But there are differences both in the signal level and in the shape of the signals corresponding to the groups combined with oxygen. It is supposed that the signal corresponding to the groups $\mathrm{CH}$ (2800$2900 \mathrm{~cm}^{-1}$ ) decreased in comparison with the CO signal (1000-1100 $\mathrm{cm}^{-1}$ ) for samples $\mathrm{Cu} 3$ and $\mathrm{Cu} 6$ in comparison with $\mathrm{Cu} 5$ sample.

This indicates that the oxygen plasma partially removed the organic layer from the substrate and left a layer containing inorganics in the form of barium and titanium. The growth of CO signals combined with oxygen ion shows an enrichment of the obtained layer in oxygen. Based on the results, it can be concluded that the obtained layers contain barium and titanium.

\section{Conclusion}

On the basis of the test results it can be stated that it is possible to obtain a layer containing titanium and barium on the copper substrate with the use of dielectric barrier discharge at atmospheric pressure. The results of analysis by the secondary ion mass spectrometry (SIMS) showed directly that both method I and method II gave positive results in the deposition of a certain amount of compounds containing barium and titanium. However, method I turned out to be more effective in obtaining 
this kind of thin layer. By contrast, the results of the analysis by FTIR allow us to indirectly draw conclusions about the contents of the required elements in the examined layers obtained by the method I. The FTIR study showed that the layer deposited on a copper substrate contained the group- $\mathrm{CO}$ combined with the oxygen connected with metal ion binding. The only metals in the solution are barium and titanium, so it can be concluded that the obtained layer had to contain these elements. FTIR study also showed that the oxygen etching in the method I resulted in decrease of the organic layer. Barium and titanium can be found on the plasma modified surface but they are dispersed in the organic phase.

This work was supported by the Tele \& Radio Research Institute Statutory Fund.

\section{References}

1. A. Purwanto, W.-N. Wang, I. Wuled Lenggoro, K. Okuyama, J. Eur. Ceram. Soc. 27, 4489 (2007)

2. V. Craciun, R.K. Singh, Appl. Phys. Lett. 76, 1932 (2000)

3. J. Gonzalo, R. Gomez San Roman, J. Perriere, C.N. Afonso, R. Perez Casero, Appl. Phys. A 66, 487 (1998)

4. K. Suzuki, K. Kijima, Vacuum 80, 519 (2006)

5. H.-H. Huang, F.-Y. Hsiao, N.-C. Wu, M.-C. Wang, J. Non-Cryst. Solids 351, 3809 (2005)

6. V. Buscaglia, M. Viviani, M.T. Buscaglia, P. Nanni, L. Mitoseriu, A. Testino, E. Stytsenko, M. Daglish, Z. Zhao, M. Nygren, Powder Technol. 148, 24 (2004)

7. W. Suchanek, T. Watanabe, M. Yoshimura, Solid State Ion. 109, 65 (1998)
8. P. Ctibor, H. Ageorges, J. Sedlacek, R. Ctvrtlik, Ceram. Int. 36, 2155 (2010)

9. K. Suzuki, K. Kijima, Mater. Lett. 58, 1650 (2004)

10. Y. Guo, K. Suzuki, K. Nishizawa, T. Miki, K. Kato, J. Cryst. Growth 284, 190 (2005)

11. J.-L. Zeng, H.-P. Teng, F.-H. Lu, Surf. Coat. Technol., DOI: $10.1016 / \mathrm{j}$. surfcoat.2011.12.049

12. F.C.M. Woudenberg, W.F.C. Sager, J.E. ten Elshof, H Verweij, Thin Solid Films 471, 134 (2005)

13. H.-H. Huang, M.-C. Wang, H.-J. Lin, M.-H. Hon, F.-Y. Hsiao, N.-C. Wu, C.-S. His, Ceram. Int. 37, 3167 (2011)

14. M. Yoshimura, W.L. Suchanek, T. Watanabe, B. Sakurai, J. Eur. Ceram. Soc. 19, 1353 (1999)

15. B. Guigues, J. Guillan, E. Defay, P. Garrec, D. Wolozan, B. Andre, F. Laugier, R. Pantel, X. Gagnard, M. Aid, J. Eur. Ceram. Soc. 27, 3851 (2007)

16. J. Herrán, G. Ga Mandayo, E. Castaño, Thin Solid Films 517, $6192(2009)$

17. S.A. Nasser, Appl. Surf. Sci. 157, 14 (2000)

18. P. Keller, H. Ferkel, K. Zweiacker, J. Naser, J.-U. Meyer, W. Riehemann, Sens. Actuators B 57, 39 (1999)

19. P. Konarski, A. Mierzejewska, Appl. Surf. Sci. 203, 354 (2003)

20. Spectral Database for Organic Compounds SDBS free site organized by National Institute of Advanced Industrial Science and Technology (AIST), Japan, http://riodb01.ibase.aist.go.jp/sdbs/ cgi-bin/cre_index.cgi

Open Access This article is distributed under the terms of the Creative Commons Attribution Noncommercial License which permits any noncommercial use, distribution, and reproduction in any medium, provided the original author(s) and source are credited. 